\title{
MC1R variants in childhood and adolescent melanoma: a retrospective pooled analysis of a multicentre cohort
}

\author{
Cristina Pellegrini*, Francesca Botta*, Daniela Massi, Claudia Martorelli, Fabio Facchetti, Sara Gandini, Patrick Maisonneuve, \\ Marie-Françoise Avril, Florence Demenais, Brigitte Bressac-de Paillerets, Veronica Hoiom, Anne E Cust, Hoda Anton-Culver, Stephen B Gruber, \\ Richard P Gallagher, Loraine Marrett, Roberto Zanetti, Terence Dwyer, Nancy E Thomas, Colin B Begg, Marianne Berwick, Susana Puig, \\ Miriam Potrony, Eduardo Nagore, Paola Ghiorzo, Chiara Menin, Ausilia Maria Manganoni, Monica Rodolfo, Sonia Brugnara, Emanuela Passoni, \\ Lidija KandolfSekulovic, Federica Baldini, Gabriella Guida, Alexandros Stratigos, Fezal Ozdemir, Fabrizio Ayala, Ricardo Fernandez-de-Misa, \\ Pietro Quaglino, Gloria Ribas, Antonella Romanini, Emilia Migliano, Ignazio Stanganelli, Peter A Kanetsky, Maria Antonietta Pizzichetta, \\ Jose Carlos García-Borrón, Hongmei Nan, Maria Teresa Landi, Julian Little, Julia Newton-Bishop, Francesco Sera, Maria Concetta Fargnoli, \\ Sara Raimondi, for the IMI Study Group, the GEM Study Group, and the M-SKIP Study Group
}

\section{Summary}

Background Germline variants in the melanocortin 1 receptor gene (MC1R) might increase the risk of childhood and adolescent melanoma, but a clear conclusion is challenging because of the low number of studies and cases. We assessed the association of $M C 1 R$ variants with childhood and adolescent melanoma in a large study comparing the prevalence of $M C 1 R$ variants in child or adolescent patients with melanoma to that in adult patients with melanoma and in healthy adult controls.

Methods In this retrospective pooled analysis, we used the M-SKIP Project, the Italian Melanoma Intergroup, and other European groups (with participants from Australia, Canada, France, Greece, Italy, the Netherlands, Serbia, Spain, Sweden, Turkey, and the USA) to assemble an international multicentre cohort. We gathered phenotypic and genetic data from children or adolescents diagnosed with sporadic single-primary cutaneous melanoma at age 20 years or younger, adult patients with sporadic single-primary cutaneous melanoma diagnosed at age 35 years or older, and healthy adult individuals as controls. We calculated odds ratios (ORs) for childhood and adolescent melanoma associated with $M C 1 R$ variants by multivariable logistic regression. Subgroup analysis was done for children aged 18 or younger and 14 years or younger.

Findings We analysed data from 233 young patients, 932 adult patients, and 932 healthy adult controls. Children and adolescents had higher odds of carrying MC1R $\mathrm{r}$ variants than did adult patients (OR 1.54, 95\% CI 1.02-2.33), including when analysis was restricted to patients aged 18 years or younger $(1 \cdot 80,1 \cdot 06-3 \cdot 07)$. All investigated variants, except Arg160Trp, tended, to varying degrees, to have higher frequencies in young patients than in adult patients, with significantly higher frequencies found for Val60Leu (OR 1.60, 95\% CI 1.05-2.44; $p=0 \cdot 04)$ and Asp294His $(2 \cdot 15,1 \cdot 05-4 \cdot 40 ; \mathrm{p}=0 \cdot 04)$. Compared with those of healthy controls, young patients with melanoma had significantly higher frequencies of any $M C 1 R$ variants.

Interpretation Our pooled analysis of $M C 1 R$ genetic data of young patients with melanoma showed that $M C 1 R \mathrm{r}$ variants were more prevalent in childhood and adolescent melanoma than in adult melanoma, especially in patients aged 18 years or younger. Our findings support the role of $M C 1 R$ in childhood and adolescent melanoma susceptibility, with a potential clinical relevance for developing early melanoma detection and preventive strategies.

Funding SPD-Pilot/Project-Award-2015; AIRC-MFAG-11831.

Copyright (C) 2019 Elsevier Ltd. All rights reserved.

\section{Introduction}

Cutaneous melanoma mainly occurs in adult patients and is rare in the paediatric population, with only $2 \%$ of all cutaneous melanoma cases diagnosed in patients younger than 20 years. $^{1-4}$ In the child and adolescent population, most cases of cutaneous melanoma are diagnosed among adolescents, with only $8 \%$ occurring in infancy and childhood., ${ }^{5,6}$

Differences exist between childhood or adolescent and adult cutaneous melanoma regarding clinical aspects, histopathological features, and disease staging., ${ }^{2,7}$
Cutaneous melanoma in childhood is often amelanotic, shows broad histopathological variability, and can present with histological uncertainty and ambiguous atypical characteristics that do not allow a definite malignant or benign classification., ${ }^{49}$ Children with cutaneous melanoma present at a more advanced stage of disease, with thicker lesions and higher rates of lymph node metastasis than do their adult counterparts, leading to a worse prognosis. ${ }^{4,9}$ However, published studies have reported discordant data on survival rates. $^{5,10}$
Lancet Child Adolesc Health 2019 Published Online March 11, 2019 http://dx.doi.org/10.1016/ S2352-4642(19)30005-7 See Online/Comment http://dx.doi.org/10.1016/ S2352-4642(19)30026-4 ${ }^{*}$ Contributed equally

Department of Dermatology and Department of Biotechnological and Applied Clinical Sciences, University of L'Aquila, L'Aquila, Italy (C Pellegrini PhD C Martorelli PhD Prof M ( Fargnoli MD); Division of Epidemiology and Biostatistics (F Botta MSC, P Maisonneuve Eng), Molecular and Pharmaco-Epidemiology Unit, Department of Experimental Oncology (S Gandini PhD, S Raimondi PhD), and Division of Melanoma, Sarcoma and Rare Cancer (F Baldini MD), European Institute of Oncology IRCCS, Milan, Italy; Department of Statistics and Quantitative Methods, University of MilanoBicocca, Milan, Italy (F Botta); Division of Pathological Anatomy, Department of Surgery and Translational Medicine, University of Florence, Florence, Italy (Prof D Massi MD); Pathology Section, Department of Molecular and Translational Medicine (Prof F Facchetti MD), and Department of Dermatology (A M Manganoni MD), Spedali Civili di Brescia, University of Brescia, Brescia, Italy; APHP, Dermatology Department, Hôpital Cochin and Paris Descartes University, Paris, France (Prof M-F Avril MD); Genetic Variation and Human Diseases Unit (UMR-946) 
Institut National de la Santé et de la Recherche Médicale (INSERM), Paris, France (Prof F Demenais MD); Department of Biopathology and INSERM, University of Paris-Saclay, Villejuif, France (B Bressac-de Paillerets PhD); Department of Oncology and Pathology, Cancer Centre, Karolinska Institutet, Stockholm, Sweden (V Hoiom PhD); Sydney School of Public Health and Melanoma Institute Australia, University

of Sydney, Sydney, NSW,

Australia (A E Cust MD); Department of Epidemiology, University of California, Irvine, CA, USA (H Anton-Culver MD); USC Norris Comprehensive Cancer Center, University of Southern California, Los Angeles, CA, USA (S B Gruber MD); British Columbia Cancer and Department of Dermatology and Skin Science, University of British Columbia, Vancouver, BC, Canada (R P Gallagher MD); Cancer Care Ontario, Toronto, ON, Canada (Prof L Marrett PhD); Piedmont Cancer Registry, Centre for Epidemiology and Prevention

in Oncology in Piedmont, Turin, Italy (R Zanetti MD); George Institute for Global

Health, Nuffield Department of Obstetrics and Gynaecology, University of Oxford, Oxford, UK (Prof T Dwyer MD): Lineberger Comprehensive Cancer Center, University of North Carolina, Chapel Hill, NC, USA (N E Thomas MD); Department of Epidemiology and Biostatistics, Memorial Sloan Kettering Cancer Center, New York, NY, USA (C B Begg PhD); Department of Internal Medicine, University

of New Mexico Cancer Center,

University of New Mexico, Albuquerque, NM, USA (Prof M Berwick MD); Melanoma Unit, Dermatology

Department, Hospital Clinic

Barcelona, University of Barcelona, Institut

$\mathrm{d}^{\prime}$ Investigacions Biomèdiques August Pi I Sunyer, and CIBER de Enfermedades Raras, Barcelona, Spain (S Puig MD, M Potrony PhD); Department of

Dermatology, Instituto Valenciano de Oncologia, Valencia, Spain (Prof E Nagore MD); Department of Internal Medicine and Medical
Research in context

\section{Evidence before this study}

The development of melanoma in children and adolescents has been hypothesised to have a stronger genetic component than that of melanoma in adults. We searched PubMed for studies published up to July 31,2018 , on melanoma susceptibility in paediatric patients, without language or date restrictions. We used the search terms "pediatric melanoma" OR "childhood melanoma" OR "adolescent melanoma" AND "susceptibility" OR "predisposition" OR "genetics". We found that genetic predisposition for melanoma has been poorly investigated in childhood and adolescence because of the rarity of the disease. Most published research included few cases, mainly from singleinstitution cohorts, investigating the main susceptibility genes for melanoma CDKN2A (cyclin-dependent kinase inhibitor 2A), CDK4 (cyclin-dependent kinase 4), and MC1R (melanocortin 1 receptor).

The overall findings reported a marginal role in paediatric patients of the two major melanoma susceptibility genes, CDKN2A and CDK4. By contrast, a high frequency of germline variants has been identified in the intermediate-penetrance MC1R gene, but the very low number of paediatric cases of melanoma made any significant conclusions impossible. Therefore, we hypothesised that a large-scale association study could explore the importance of the MC1R gene in paediatric melanoma predisposition.

Whether adult melanoma and childhood and adolescent melanoma share a similar pathogenesis has long been a subject of debate. Major risk factors for paediatric cutaneous melanoma include giant congenital melanocytic naevi and hereditary conditions such as xeroderma pigmentosum, immunodeficiency, and albinism. ${ }^{11}$ Other known risk factors common to paediatric and adult melanoma are family history of melanoma, dysplastic naevus syndrome, elevated number of acquired melanocytic naevi, red hair, sun-sensitive phenotype, and ultraviolet radiation (UV) exposure. . $^{12,13}$

It is uncertain whether childhood and adolescent cutaneous melanoma differs from the adult melanoma regarding genetic predisposition. The paediatric form of the disease is mostly sporadic, whereas adolescent cutaneous melanoma is sometimes observed in melanoma-prone families. In general, there is a higher proportion of germline mutation carriers among young patients with cancer than among older patients, ${ }^{14}$ but whether this tendency holds true for cutaneous melanoma is unclear because of the rarity of this disease occurring in children or adolescents. On the basis of the few available studies, ${ }^{12,15-21}$ child and adolescent patients have only rarely been found to carry germline mutations in the two high-penetrance melanoma genes, CDKN2A and $C D K 4$, which are known to be significantly associated with melanoma in a familial context alone.

The MC1R (melanocortin 1 receptor) gene is a key determinant of human pigmentation..$^{22} M C 1 R$ is highly

\section{Added value of this study}

Our study assessed the effect of MC1R gene variants on paediatric melanoma susceptibility in a large case-case study, by comparing the prevalence of $M C 1 R$ variants in child or adolescent patients with those in adult patients and in healthy controls. To our knowledge, our series of patients is the largest international multicentre cohort of paediatric patients with melanoma with available genetic data. Our pooled analysis showed that paediatric patients had a higher probability of carrying any MC1R variant than that of adult patients, suggesting a major role of MC1R variants, mainly $r$ variants, in paediatric melanoma predisposition. Furthermore, $r$ variants seemed to be most strongly associated with melanoma in patients aged 18 years or younger.

\section{Implications of all the available evidence}

We provided evidence of genetic determinants potentially involved in paediatric melanoma susceptibility. Our study represents a first step to comprehend the genetic background of paediatric melanoma and to elucidate the diversity of paediatric and adult melanoma, with potential clinical implications. polymorphic in the general population, and specific variants were defined as $\mathrm{R}$ (Asp84Glu, Arg142His, Arg151Cys, Ile155Thr, Arg160Trp, Asp294His) or $r$ (Val60Leu, Val92Met, Arg163Gln) alleles, according to their strength of association with the red hair colour phenotype. ${ }^{23}$ Extensive in vitro and in vivo evidence showed that both $\mathrm{R}$ and $\mathrm{r}$ alleles produce hypomorphic proteins with compromised activity compared with native MC1R function. ${ }^{22}$ The $\mathrm{R}$ alleles have been found to have a major effect on pigmentation and UV sensitivity. ${ }^{22,23}$ By contrast, $r$ alleles confer normal or slightly impaired MC1R activity, resulting in a low-strength association with the fair skin phenotype. ${ }^{23}$

Natural $M C 1 R$ variation is an established risk factor for cutaneous melanoma across multiple populations worldwide. ${ }^{24}$ The risk of cutaneous melanoma is higher for carriers of an $M C 1 R$ variant than for wild-type individuals, with the strongest association among carriers of $\mathrm{R}$ alleles and multiple variants. ${ }^{24} M C 1 R$ variants confer a significant increased risk of cutaneous melanoma in darkly pigmented individuals, highlighting the effect of $M C 1 R$ through non-pigmentary pathways. ${ }^{25,26}$ Moreover, $M C 1 R$ variant genotypes are associated with phenotypic characteristics of melanoma ${ }^{27}$ and melanocytic naevi ${ }^{28}$ and seem to influence the somatic mutational load in adult cutaneous melanoma. ${ }^{29}$ Young patients (aged 20 years or younger) with cutaneous melanoma have an elevated prevalence of $M C 1 R$ variants, but the low number of available studies, coupled with the small 
number of cases per study, makes drawing clear conclusions a challenge. ${ }^{18-20}$

To help elucidate the role of MC1R in childhood and adolescent cutaneous melanoma and to better understand the genetic and clinical diversity of the disease across age, with potential clinical effects in terms of early melanoma detection and preventive strategies, we assessed these tumours in a large multicentre cohort pooled from the M-SKIP (melanocortin 1 receptor skin cancer and phenotypic characteristics) Project, the Italian Melanoma Intergroup (IMI), and other European groups. The aims of our study were to compare the prevalence of $M C 1 R$ variants between young patients and healthy controls, with a case-control study design, and between young patients and adult patients, with a case-case study design.

\section{Methods}

\section{Study design and participants}

We analysed a large, multicentre cohort pooled from the M-SKIP Project, the IMI, and other European groups (appendix), including participants from 11 countries (Australia, Canada, France, Greece, Italy, the Netherlands, Serbia, Spain, Sweden, Turkey, and the USA; figure 1). Our analysis included children and adolescents diagnosed with sporadic single-primary cutaneous melanoma at age 20 years or younger, adult patients with sporadic single-primary cutaneous melanoma diagnosed at age 35 years or older, and healthy adult individuals as controls. Because age is a continuous variable, and an exact age cutoff between adolescents and adults would not be expected, we excluded melanoma cases diagnosed in the age range of 21-34 years to avoid a possible overlap between categories, and thus enable comparison between groups with distinct clinical and genetic characteristics. Because of the known challenges in diagnosing paediatric melanoma ${ }^{30-32}$ and to decrease misdiagnosis, participating investigators were asked to provide the original histopathological reports and representative glass slides for central review. Only patients for whom the original histopathological report was available were eligible. Additionally, we restricted the study to cases with complete MC1R genotyping. We excluded familial melanoma cases, cases with a history of cancer at any site other than non-melanoma skin cancer, atypical spitzoid neoplasms or melanocytic tumors of uncertain malignant potential, and ocular and mucosal melanomas.

Detailed information on recruitment is reported in the appendix. Ethics committee approval was obtained at each institution in which new blood samples were drawn. For each young patient, four adult patients and four healthy controls were randomly selected from the same parent study that provided the young patient. When this was not possible, adult patients and controls were selected from a study that was done in the nearest geographical proximity to the parent study of the young patient (appendix; figure 1). Written consent was obtained from adult and

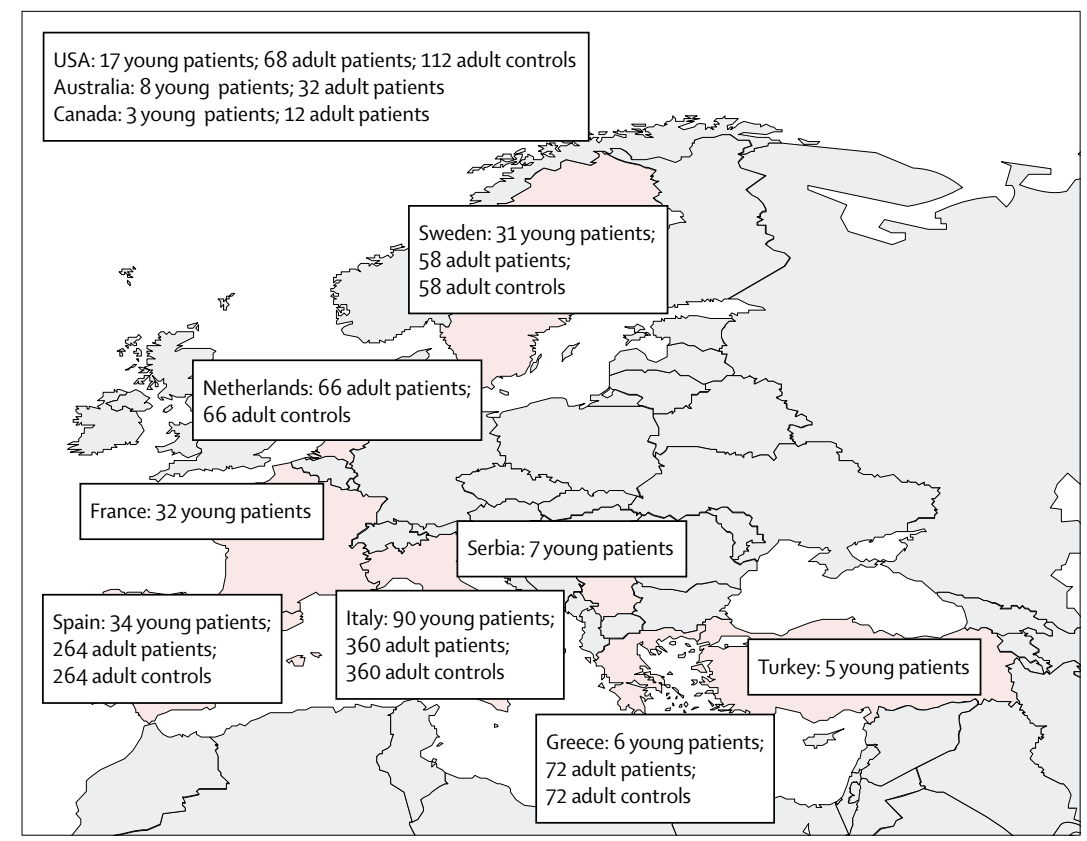

Figure 1: Geographical areas of participant recruitment

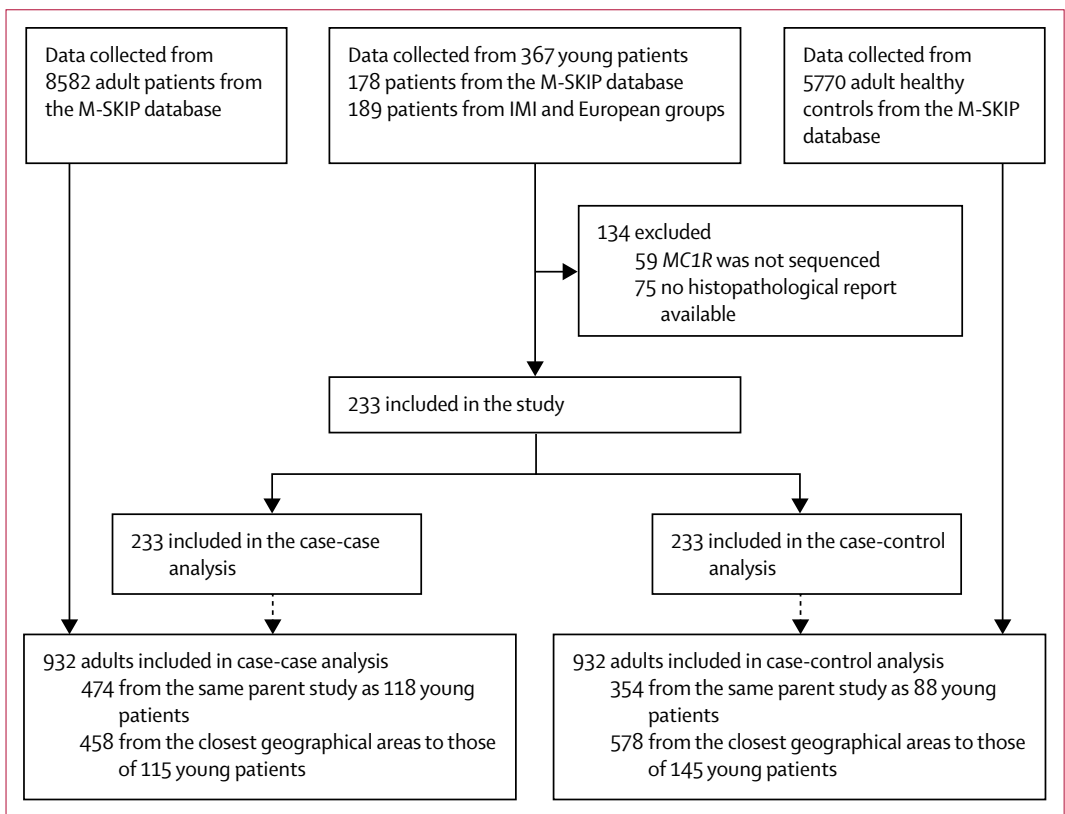

Figure 2: Flow chart of participants included in the analysis

Young patients were aged 20 years or younger, whereas adult patients and healthy controls were aged 35 years or older. M-SKIP=melanocortin 1 receptor skin cancer and phenotypic characteristics Project. IMI=Italian Melanoma Intergroup. $M C 1 R=$ melanocortin 1 receptor.

older adolescent patients and the parents of young patients.

\section{Procedures}

For 135 young patients from the M-SKIP Project, and 48 from the IMI and European groups, $M C 1 R$ sequencing had already been done in study-specific laboratories
Specialties, University of Genoa and Ospedale Policlinico San Martino, Genoa, Italy (Prof P Ghiorzo PhD); Diagnostic Immunology and Molecular Oncology Unit, Veneto Institute of Oncology, IOV-IRCCS, Padua, Italy 


\begin{tabular}{|c|c|c|c|c|c|c|}
\hline $\begin{array}{r}\text { (C Menin MD); Department of } \\
\text { Research, Fondazione IRCCS }\end{array}$ & & Young patients $(n=233$ ) & Adult patients $(n=932)$ & p value ${ }^{*}$ & Adult controls $(n=932)$ & p value* \\
\hline Istituto Nazionale dei Tumori, & Sex & .. & .. & 0.025 & .. & 0.00030 \\
\hline Milan, Italy (M Rodolfo MSc); & Male & $95(41 \%)$ & $456(49 \% ; n=931)$ &.$\cdot$ & $500(54 \% ; n=926)$ &.. \\
\hline Hospital, Trento, Italy & Female & $138(59 \%)$ & $475(51 \% ; n=931)$ &. & $426(46 \% ; n=926)$ & .. \\
\hline (S Brugnara MD); Department & Median Breslow thickness, mm (IQR) & $0.93(0.50-2.10)$ & $1 \cdot 00(0 \cdot 50-2 \cdot 40)$ & $0 \cdot 16$ & NA & NA \\
\hline of Pathophysiology and & Median common melanocytic naevi, count (IQR) & $30(15-64)$ & $25(10-45)$ & 0.00070 & $21(5-30)$ & $<0.0001$ \\
\hline $\begin{array}{l}\text { Transplantation, University of } \\
\text { Milan, Foundation IRCCS Ca' }\end{array}$ & Any atypical melanocytic naevi & $49(43 \% ; n=113)$ & $165(30 \% ; n=556)$ & 0.010 & $46(9 \% ; n=501)$ & $<0.0001$ \\
\hline Granda Ospedale Maggiore & Melanoma body site & .. & .. & 0.037 & .. & NA \\
\hline Policlinico, Milan, Italy & Head or neck & $27(12 \% ; n=222)$ & $127(16 \% ; n=808)$ & .. & NA & .. \\
\hline (E Passoni MD); Department of & Trunk & $91(41 \% ; n=222)$ & $313(39 \% ; n=808)$ &. & NA &. \\
\hline $\begin{array}{l}\text { Dermatology, racuity of } \\
\text { Medicine, Military Medical }\end{array}$ & Upper limbs & $11(5 \% ; n=222)$ & $90(11 \% ; n=808)$ &. & NA &. \\
\hline Academy, Belgrade, Serbia & Lower limbs & $75(34 \% ; n=222)$ & $236(29 \% ; n=808)$ & .. & NA & .. \\
\hline (Prof L K Sekulovic MD); & NOC + & $18(8 \% ; n=222)$ & $42(5 \% ; n=808)$ &.. & NA &. \\
\hline Sciences, Neurosciences and & Histolopathogical subtype & .. & .. & $<0.0001$ &.. & NA \\
\hline Sense Organs, University of & LMM & $0(0 \%)$ & $50(7 \% ; n=719)$ &.. & NA & .. \\
\hline Bari Aldo Moro, Bari, Italy & NM & $33(17 \% ; n=198)$ & $127(18 \% ; n=719)$ &.. & NA &. \\
\hline (G Guida PhD); 1st Department & SSM & $124(63 \% ; n=198)$ & $493(69 \% ; n=719)$ &. & NA & .. \\
\hline $\begin{array}{l}\text { of Dermatology, Andreas } \\
\text { Sygros Hospital, Medical }\end{array}$ & ALM & $7(3 \% ; n=198)$ & $39(5 \% ; n=719)$ &. & NA &. \\
\hline School, National and & Spitzoid & $13(7 \% ; n=198)$ & $2(<1 \% ; n=719)$ & .. & NA &.. \\
\hline Kapodistrian University of & Others $\ddagger$ & $21(11 \% ; n=198)$ & $8(1 \% ; n=719)$ &.$\cdot$ & NA &. \\
\hline $\begin{array}{l}\text { Athens, Athens, Greece } \\
\text { (Prof A Stratigos MD); }\end{array}$ & Hair colour & .. & .. & $0 \cdot 73$ &.. & 0.00030 \\
\hline Department of Dermatology, & Red & $14(7 \% ; n=213)$ & $55(6 \% ; n=895)$ &.. & $24(3 \% ; n=709)$ &.$\cdot$ \\
\hline Faculty of Medicine, University & Blonde & $60(28 \% ; n=213)$ & $216(24 \% ; n=895)$ & .. & $129(18 \% ; n=709)$ &.. \\
\hline $\begin{array}{r}\text { of Ege, Izmir, Turkey } \\
\text { (Prof F Ozdemir MD); Melanoma }\end{array}$ & Brown & $139(65 \% ; n=213)$ & $609(68 \% ; n=895)$ &. & $535(76 \% ; n=709)$ &.. \\
\hline Unit, Cancer Immunotherapy & NOCt & $0(0 \%)$ & $15(2 \% ; n=895)$ &.. & $21(3 \% ; n=709)$ &. \\
\hline and Innovative Therapies, & Eye colour &.. & .. & 0.011 &.. & $<0.0001$ \\
\hline IRCCS Istituto Nazionale dei & Blue & $65(36 \% ; n=181)$ & $420(50 \% ; n=845)$ & .. & $330(47 \% ; n=709)$ &.. \\
\hline $\begin{array}{r}\text { Tumori, Fondazione G Pascale, } \\
\text { Napoli, Italia (F Ayala MD); }\end{array}$ & Brown & $77(42 \% ; n=181)$ & $314(37 \% ; n=845)$ & .. & $364(51 \% ; n=709)$ & .. \\
\hline Dermatology Service, & Black & $2(1 \% ; n=181)$ & $2(<1 \% ; n=845)$ &.. & $5(1 \% ; n=709)$ & .. \\
\hline University Hospital Nuestra & Green, grey, hazel & $5(3 \% ; n=181)$ & $0(0 \%)$ &.. & $0(0 \%)$ &.. \\
\hline $\begin{array}{r}\text { Senora de Candelaria, } \\
\text { Santa Cruz de Tenerife, Spain }\end{array}$ & $\mathrm{NOC} \dagger$ & $32(18 \% ; n=181)$ & $109(13 \% ; n=845)$ &. & $10(1 \% ; n=709)$ & .. \\
\hline (R Fernandez-de-Misa PhD); & Skin type & .. & .. & 0.67 & .. & 0.015 \\
\hline Dermatologic Clinic, & 1 & $16(8 \% ; n=210)$ & $59(7 \% ; n=838)$ &.. & $26(4 \% ; n=682)$ &.. \\
\hline Department of Medical & ॥ & $68(33 \% ; n=210)$ & $320(36 \% ; n=838)$ & .. & $191(28 \% ; n=682)$ &. \\
\hline $\begin{array}{l}\text { Sciences, University of Torino, } \\
\text { Turin, Italy (P Quaglino MD); }\end{array}$ & III & $94(44 \% ; n=210)$ & $400(45 \% ; n=838)$ &. & $378(55 \% ; n=682)$ &. \\
\hline Department of Medical & IV & $32(15 \% ; n=210)$ & $59(13 \% ; n=838)$ &.$\cdot$ & $87(13 \% ; n=682)$ &. \\
\hline $\begin{array}{l}\text { Oncology and Haematology, } \\
\text { Fundación Investigación }\end{array}$ & Any solar lentigines & $15(15 \% ; n=100)$ & $321(75 \% ; n=428)$ & $<0.0001$ & $203(68 \% ; n=299)$ & $<0.0001$ \\
\hline $\begin{array}{r}\text { Clínico de Valencia, INCLIVA } \\
\text { Instituto de Investigación } \\
\text { Sanitaria, Valencia, Spain } \\
\text { (G Ribas PhD); US Ambulatori } \\
\text { Melanomi, Sarcomi e Tumori }\end{array}$ & \multicolumn{6}{|c|}{$\begin{array}{l}\text { Data are } n(\%) \text {, unless otherwise specified. } N A=\text { not applicable. } N O C=\text { not otherwise classifiable. } L M M=l \text { entigo maligna melanoma. NM=nodular melanoma. SSM=superficial } \\
\text { spreading melanoma. ALM=acral lentiginous melanoma. *Logistic regression model, adjusted by matching stratum variable. } † \text { This group includes patients with doubtful or } \\
\text { mixed information, thus not classifiable. ‡Other subtypes among children or adolescents include nevoid }(n=4) \text {, epithelioid }(n=3) \text {, desmoplastic ( } n=1) \text {, and others not specified } \\
(n=13) \text {; subtypes among adults include epithelioid }(n=5) \text {, nevoid }(n=1) \text {, desmoplastic }(n=1) \text {, and others not specified }(n=1) \text {. }\end{array}$} \\
\hline Rari, UO Oncologia Medica 1, & Table 1: Characteristics of the study population & & & & & \\
\hline
\end{tabular}

Universitaria Santa Chiara,

Pisa, Italy (A Romanini MD);

Plastic Surgery, San Gallicano Dermatological Institute, IRCCS, Rome, Italy

(E Migliano MD); Skin Cancer Unit, IRCCS Scientific Institute of Romagna for the Study and Treatment of Cancer and University of Parma, Meldola, Italy (IStanganelli MD); Department of Cancer Epidemiology, H Lee Moffitt Cancer Center and Research Institute, Tampa, FL, USA (appendix). For the remaining 50 young patients from IMI and European groups who provided new blood or saliva samples, $M C 1 R$ genotyping was done centrally at the University of L'Aquila (L'Aquila, Italy) and done as described elsewhere. ${ }^{33}$

\section{Statistical analysis}

A complete description of the statistical analysis is available in the appendix. Briefly, we analysed the associations between risk factors and young melanoma by logistic regression in comparison with two reference groups, adult patients and healthy controls, with adjustment for study or geographical location.

We compared the frequency of any $M C 1 R$ variants among children or adolescents with that of adult patients and controls by logistic regression, with adjustment for study or geographical location. These comparisons were repeated for any $M C 1 R \mathrm{R}$ variant, for any $\mathrm{r}$ variant, for a score calculated by summing across the $M C 1 R$ alleles, which gives a value of 1 to $r$ and 2 to $R$ variants (as proposed elsewhere), ${ }^{34}$ and for each of the nine most prevalent $M C 1 R$ variants and any rare $M C 1 R$ variants (presence or absence). 


\begin{tabular}{|c|c|c|c|c|c|c|c|c|c|c|}
\hline & \multicolumn{5}{|c|}{ All studied patients } & \multicolumn{5}{|c|}{ Patients with centralised confirmed melanoma diagnosis } \\
\hline & $\begin{array}{l}\text { Young patients } \\
(\mathrm{n}=233)\end{array}$ & $\begin{array}{l}\text { Adult patients } \\
(\mathrm{n}=932)\end{array}$ & p value* & $\begin{array}{l}\text { Adult controls } \\
(\mathrm{n}=932)\end{array}$ & p value* & $\begin{array}{l}\text { Young patients } \\
(\mathrm{n}=64)\end{array}$ & $\begin{array}{l}\text { Adult patients } \\
(\mathrm{n}=256)\end{array}$ & p value* & $\begin{array}{l}\text { Adult controls } \\
(\mathrm{n}=256)\end{array}$ & p value* \\
\hline Any MC1R variants & $173(74 \%)$ & $662(71 \%)$ & 0.33 & $550(59 \%)$ & $<0.0001$ & $46(72 \%)$ & $193(75 \%)$ & 0.56 & $145(57 \%)$ & 0.028 \\
\hline Any R variants & $86(37 \%)$ & $350(38 \%)$ & 0.86 & $238(26 \%)$ & 0.00060 & $24(38 \%)$ & $102(40 \%)$ & 0.73 & $58(23 \%)$ & 0.016 \\
\hline Any rvariants & $115(49 \%)$ & $420(45 \%)$ & 0.24 & $370(40 \%)$ & 0.0077 & $29(45 \%)$ & $115(45 \%)$ & 0.95 & $102(40 \%)$ & 0.43 \\
\hline Score &.. &.. & 0.85 & .. & $<0.0001$ & .. & .. & 0.38 & .. & 0.0029 \\
\hline 0 & $60(26 \%)$ & $270(29 \%)$ &.. & $382(41 \%)$ &. & $18(28 \%)$ & $63(25 \%)$ &.. & $111(43 \%)$ & .. \\
\hline 1 & $71(30 \%)$ & $260(28 \%)$ &. & $261(28 \%)$ &. & $16(25 \%)$ & $70(27 \%)$ &.. & $77(30 \%)$ &.. \\
\hline 2 & $64(27 \%)$ & $227(24 \%)$ &. & $201(22 \%)$ &.. & $20(31 \%)$ & $72(28 \%)$ &.. & $47(18 \%)$ & .. \\
\hline 3 & $28(12 \%)$ & $106(11 \%)$ &.. & $57(6 \%)$ &.. & $7(11 \%)$ & $24(9 \%)$ &.. & $15(6 \%)$ &.. \\
\hline$\geq 4$ & $10(4 \%)$ & $69(7 \%)$ &. & $31(3 \%)$ & .. & $3(5 \%)$ & $27(11 \%)$ &. & $6(2 \%)$ & .. \\
\hline Any Val6oLeu variants & $77(33 \%)$ & $270(29 \%)$ & 0.22 & $251(27 \%)$ & 0.060 & $24(38 \%)$ & $82(32 \%)$ & 0.40 & $70(27 \%)$ & 0.11 \\
\hline Any Asp84Glu variants & $3(1 \%)$ & $14(2 \%)$ & 0.81 & $7(1 \%)$ & 0.43 & $1(2 \%)$ & $3(1 \%)$ & 0.80 & $1(0 \%)$ & 0.32 \\
\hline Any Val92Met variants & $30(13 \%)$ & $115(12 \%)$ & 0.82 & $115(12 \%)$ & 0.83 & $9(14 \%)$ & $25(10 \%)$ & 0.32 & $29(11 \%)$ & 0.55 \\
\hline Any Arg142His variants & $7(3 \%)$ & $34(4 \%)$ & 0.63 & $22(2 \%)$ & 0.57 & $0(0 \%)$ & $12(5 \%)$ & 0.98 & $11(4 \%)$ & 0.98 \\
\hline Any Arg151Cys variants & $30(13 \%)$ & $142(15 \%)$ & 0.36 & $91(10 \%)$ & 0.17 & $11(17 \%)$ & $45(18 \%)$ & 0.94 & $23(9 \%)$ & 0.060 \\
\hline Any lle155Thr variants & $4(2 \%)$ & $18(2 \%)$ & 0.83 & $15(2 \%)$ & 0.91 & $1(2 \%)$ & $5(2 \%)$ & 0.84 & $2(1 \%)$ & 0.57 \\
\hline Any Arg160Trp variants & $21(9 \%)$ & $93(10 \%)$ & 0.66 & $63(7 \%)$ & 0.23 & $7(11 \%)$ & $29(11 \%)$ & 0.92 & $15(6 \%)$ & 0.16 \\
\hline Any Arg $163 \mathrm{Gln}$ variants & $13(6 \%)$ & $59(6 \%)$ & 0.67 & $34(4 \%)$ & 0.18 & $0(0 \%)$ & $17(7 \%)$ & 0.97 & $7(3 \%)$ & 0.98 \\
\hline Any Asp294His variants & $19(8 \%)$ & $54(6 \%)$ & 0.18 & $37(4 \%)$ & 0.0089 & $4(6 \%)$ & $17(7 \%)$ & 0.91 & $8(3 \%)$ & 0.25 \\
\hline \multicolumn{11}{|c|}{ 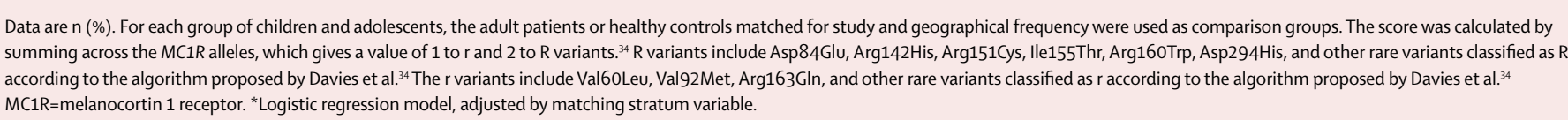 } \\
\hline
\end{tabular}

We then used multivariable unconditional logistic regression models to calculate the odds ratio (OR) for $M C 1 R$ variants after adjusting for study or geographical location and other covariables (as available) including sex, melanoma body site, histopathological subtype, hair colour, and skin type. We also did a sensitivity analysis with multivariable conditional logistic regression models. Because of the retrospective and multicentre nature of the study, information on covariables was not available for all the patients. Covariables with more than $30 \%$ of missing data were not included in the models, whereas multiple imputation models were done for variables with less than $30 \%$ of missing data (appendix).

The primary analysis compared the entire sample of young patients with adult controls and adult patients. Considering the possible misdiagnosis in young patients, we repeated the primary analysis including only the subgroup of young patients with cutaneous melanoma diagnosis confirmed after central slide review. We then calculated a modified OR, applying the method proposed by Manfred Green ${ }^{35}$ that incorporates adjustment based on the predictive value of a positive test. We also did sensitivity analyses on the subgroup of young and adult patients coming from the same parental study and on the overall sample after the exclusion of patients without confirmed diagnosis. Subgroup analyses were done according to age at diagnosis of young patients.
Generally, p values lower than 0.05 were considered statistically significant. However, we also calculated $\mathrm{p}$ values corrected for false discovery rate (FDR) to take into account multiple comparisons. We used SAS software (version 9.4) and STATA (version 15) for our analyses.

\section{Role of the funding source}

The funder of the study had no role in study design, data collection, data analysis, data interpretation, or writing of the report. The corresponding author had full access to all the data in the study and had final responsibility for the decision to submit for publication.

\section{Results}

We retrospectively collected data up to Dec 31, 2016, of 367 young patients, 8582 adult patients, and 5770 adult controls (figure 2). For 59 young patients, information on $M C 1 R$ was not available either because of patients' death (two patients) or refusal to participate in the study ( $n=57)$. Among the remaining 308 patients, 75 had no original histopathological report available, leaving 233 young patients for inclusion in the statistical analysis. For the selected 932 adult patients, 474 were from the same parent study as the young patients and 458 came from a geographically close study population. For the selected 932 adult controls, 354 were from the same parent study
(Prof PA Kanetsky PhD); University of Trieste, Centro di Riferimento Oncologico, IRCCS, Aviano, Italy

(M A Pizzichetta MD) Department of Biochemistry, Molecular Biology, and Immunology, University of Murcia and IMIB-Arrixaca, Murcia, Spain

(Prof J C García-Borrón PhD); Department of Epidemiology Richard M Fairbanks School of Public Health, Melvin \& Bren Simon Cancer Center, Indiana University, Indianapolis, IN, USA (H Nan MD); Division of Cancer Epidemiology and Genetics, National Cancer Institute, National Institutes of Health, Bethesda, MD, USA (MT Landi PhD); School of Epidemiology and Public Health, University of Ottawa, Ottawa, ON, Canada (Prof J Little PhD); Section of Epidemiology and Biostatistics, Institute of Medical Research at St James', University of Leeds, Leeds, UK (Prof J Newton-Bishop PhD); and Department of Public Health, Environments and Society, 


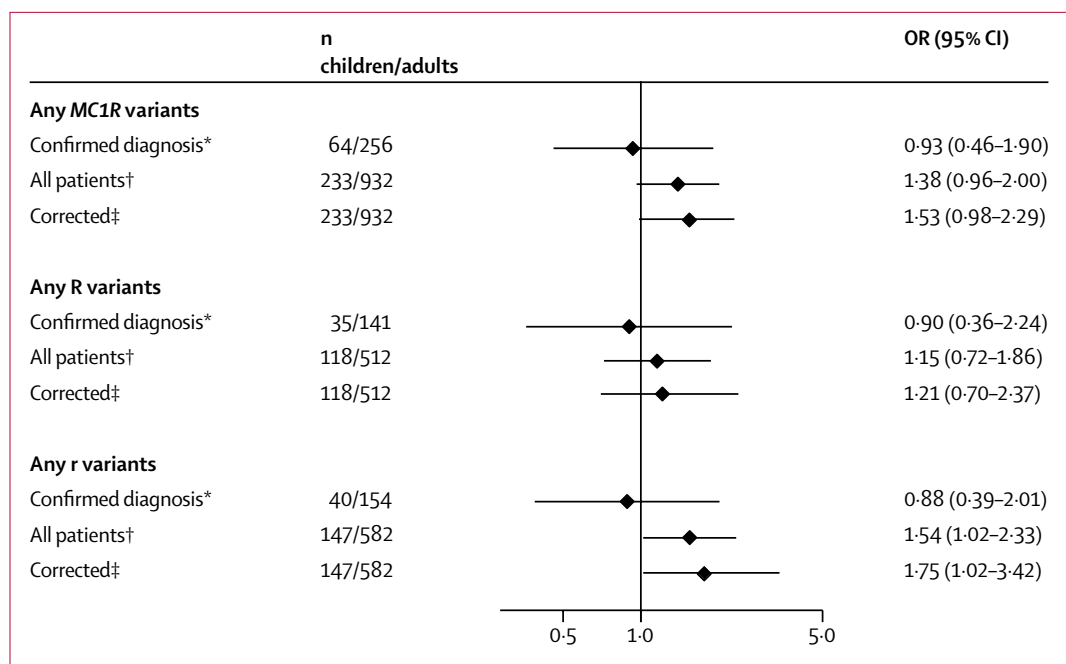

Figure 3: Covariable-adjusted odds ratio (OR) for the association between any $M C 1 R$ variants, $R$ variants, and $r$ variants and childhood and adolescent melanoma compared with adult melanoma

All ORs were adjusted by sex, matching stratum variable, melanoma body site and histopathological subtype, hair colour, and skin type. For each OR, the comparison groups included child and adolescent patients matched (4:1) with adult patients by study or geographical area. The reference category for OR was MC1R wild-type (WT) individuals. Numbers of children or adolescents and adults reported here are the total numbers of patients included in each analysis, independently by MC1R status. For the analysis on any R variant versus WT, patients carrying only $r$ variants were excluded, and for the analysis on any $r$ variant versus WT, patients carrying only $R$ variants were excluded. $R$ variants include Asp84Glu, Arg142His, Arg151Cys, lle155Thr, Arg160Trp, Asp294His, and other rare variants classified as $\mathrm{R}$ according to the algorithm proposed by Davies and colleagues. ${ }^{34}$ The r variants include Val60Leu, Val92Met, Arg163Gln, and other rare variants classified as r according to the algorithm proposed by Davies and colleagues. ${ }^{34} \mathrm{MC} 1 \mathrm{R}=$ melanocortin 1 receptor. ${ }^{*}$ Calculated on the subgroup of patients with confirmed diagnosis of melanoma after centralised pathological review of glass slides. †Calculated on the whole sample of 233 child and adolescent patients. $\neq$ Corrected for probability of misdiagnosis, by combining information from OR of confirmed diagnoses and OR of all patients, as suggested elsewhere. ${ }^{35}$

London School of Hygiene \& Tropical Medicine, London, UK (F Sera MSc)

Correspondence to: Dr Sara Raimondi, Division of Epidemiology and Biostatistics, European Institute of Oncology IRCCS, 20141 Milan, Italy sara.raimondi@ieo.it See Online for appendix as the young patients and 578 came from a geographically close study population. Young patients had a median age of 18 years (IQR 15-19), adult patients had a median age of 55 years (45-67), and adult healthy controls had a median age of 50 years (43-59). The total count of common melanocytic naevi was higher in young patients than in either adult patients or controls (table 1). Young patients had a higher proportion of atypical melanocytic naevi than those of adult patients and adult controls. We found differences between young patients and adult patients regarding the histopathological subtype of melanomas and the body site where they occurred. Children and adolescents had a lower prevalence of blue eyes than that of adult patients or controls, and they were less likely to have solar lentigines than adult patients or controls (table 1).

Table 2 shows the frequencies of any $M C 1 R$ variants, any $\mathrm{R}$ variants, any $\mathrm{r}$ variants, $M C 1 R$ score, and any of the nine most prevalent $M C 1 R$ variants in the young patients, adult patients, and adult controls in our study. With a univariable analysis, we found no significant differences in frequency of $M C 1 R$ variants between young and adult patients. However, young patients had significantly higher frequencies of any variants, $R$ variants, $r$ variants, and MC1R score than those of healthy controls, supporting the role of $M C 1 R$ in melanoma susceptibility. We found eight rare $M C 1 R$ variants in young patients: 86insA (two patients), Val51Ala, Thr95Met, Val122Met, Arg151His, Ala218Thr, Phe258Leu, Lys278Glu, (one patient each). No association was found between childhood and adolescent melanoma and any $M C 1 R$ rare variant (data not shown).

Among the 233 young patients in our cohort, representative histopathological slides of the tumour were available for 85 patients and were centrally reviewed for quality control by a dermatopathologist (DM). These 85 patients had clinicopathological characteristics similar to those of 148 patients for whom glass slides were not reviewed (appendix). The original diagnosis of melanoma was confirmed in 64 (75\%) of 85 patients. The samples of the other $21(25 \%)$ patients were deemed not representative, were difficult to interpret for technical reasons, or were reclassified as atypical melanocytic naevi, atypical junctional melanocytic proliferations, pagetoid melanocytosis overlying congenital naevi, or ambiguous atypical melanocytic proliferations with spitzoid features. In the reclassified cases, serial unstained slides or paraffin blocks were not available, and thus additional immunohistochemical or molecular analyses, which would have clarified interpretation, were precluded. Such doubtful cases were independently reviewed by a second dermatopathologist (FF), but the conflicting discrepancy with the original diagnosis remained unresolved. The median Breslow thickness was $1.00 \mathrm{~mm}$ (IQR $0 \cdot 50-1.90$ ) in the 64 patients with a confirmed diagnosis and $0.45 \mathrm{~mm}$ $(0 \cdot 10-0 \cdot 75)$ in the 21 patients for whom the original diagnosis was not confirmed ( $\mathrm{p}=0 \cdot 0005$; appendix). No other clinicopathological features differed between the two groups (appendix).

The frequencies of $M C 1 R$ variants in this subgroup of 64 children or adolescents with a confirmed diagnosis after histopathological review and in the matched 256 adult patients, and 256 controls were similar to those reported for the primary analysis (table 2).

We found that children or adolescents with cutaneous melanoma had significantly higher odds of carrying any $\mathrm{r}$ variants than those of adult patients (OR 1.54, 95\% CI $1 \cdot 02-2 \cdot 33$; FDR-corrected $\mathrm{p}=0 \cdot 17$; figure 3 ). Concerning specific $M C 1 R$ variants, we found a positive association for all $M C 1 R$ variants with childhood and adolescent melanoma, except for the Arg160Trp variant (figure 4). We found a statistically significant association for Val60Leu ( $\mathrm{p}=0 \cdot 04$, FDR-corrected $\mathrm{p}=0 \cdot 17)$ and Asp294His $(\mathrm{p}=0.04$, FDR-corrected $\mathrm{p}=0 \cdot 17$ ) variants in both the primary analysis and after correction for possible misdiagnosis (figure 4). Similar results were obtained in the sensitivity analysis with conditional logistic regression models (appendix) and by excluding the 21 children and adolescents without centrally confirmed diagnosis (appendix). Finally, when we repeated the primary analysis on the subgroups of young patients and adult patients from the same parental study, we obtained stronger associations with childhood and adolescent 
melanoma than those of the primary analysis for carriers of any $M C 1 R$ variant (OR 2.04, 95\% CI $1 \cdot 19-3 \cdot 50)$, r variants $(2 \cdot 61,1 \cdot 43-4 \cdot 73)$, and Val60Leu (2.67, 1.44-4.95) and Asp294His variants (3.12, 1.08-9.03; appendix).

We did a subgroup analysis by age at diagnosis (patients who were 18 years or younger and patients who were 14 years or younger at diagnosis), and we observed a significantly higher frequency of $\mathrm{r}$ variants in patients aged 18 years or younger than in adult patients (OR 1.80 , 95\% CI 1.06-3.07; FDR-corrected $\mathrm{p}=0 \cdot 61$; table 3 ). The corresponding OR for patients aged 14 years or younger was even higher, but was not significant because of the small number of patients.

We also did a case-control analysis comparing young patients with melanoma with healthy adult controls (appendix). We found a significantly higher risk of childhood and adolescent melanoma in carriers of any $M C 1 R, \mathrm{R}$, and $\mathrm{r}$ variants, as well as for the most common MC1R Val60Leu, Val92Met, Arg151Cys, Arg163Gln, and Asp294His variants compared with that in healthy controls. Results remained significant after correction for multiple comparison, except for the Val92Met variant (FDR-corrected $\mathrm{p}=0 \cdot 07)$.

\section{Discussion}

Our pooled-analysis showed that young patients had significantly higher frequencies of any $M C 1 R$ variants, $R$ variants, and $r$ variants than those of healthy controls, supporting the role of $M C 1 R$ variants as genetic risk factors for childhood and adolescent cutaneous melanoma. We also found that the frequency of $r$ variants was elevated in young patients compared with that of adult patients. The effect of $r$ variants was supported by analyses limited to individuals aged 18 years or younger and was even stronger, but not significantly, for children aged 14 years or younger, suggesting a higher prevalence of $M C 1 R$ variants in childhood melanoma. The $M C 1 R$ Val60Leu and Asp294His variants showed the most robust association with melanoma in childhood and adolescence, even after correction for possible misdiagnosis.

Childhood and adolescent melanoma has been reported to occur most commonly in white people and in girls. ${ }^{2,10,13}$ In line with two previous studies, ${ }^{12,13}$ we found that young patients with melanoma are characterised by a fairer phenotype than that of healthy controls, including traits such as red hair and skin type. By contrast, young patients presented with more darkly pigmented characteristics, such as brown eyes, skin type III or IV, and a lower prevalence of freckles compared with those of location-matched adult patients. Consistent with most published studies, ${ }^{2,11,36}$ our young patients showed a high number of melanocytic naevi, both common and atypical, and developed melanomas mainly on the lower limbs and the trunk. Childhood and adolescent melanoma was more commonly diagnosed as nodular melanoma compared with its adult counterpart. Spitzoid melanomas

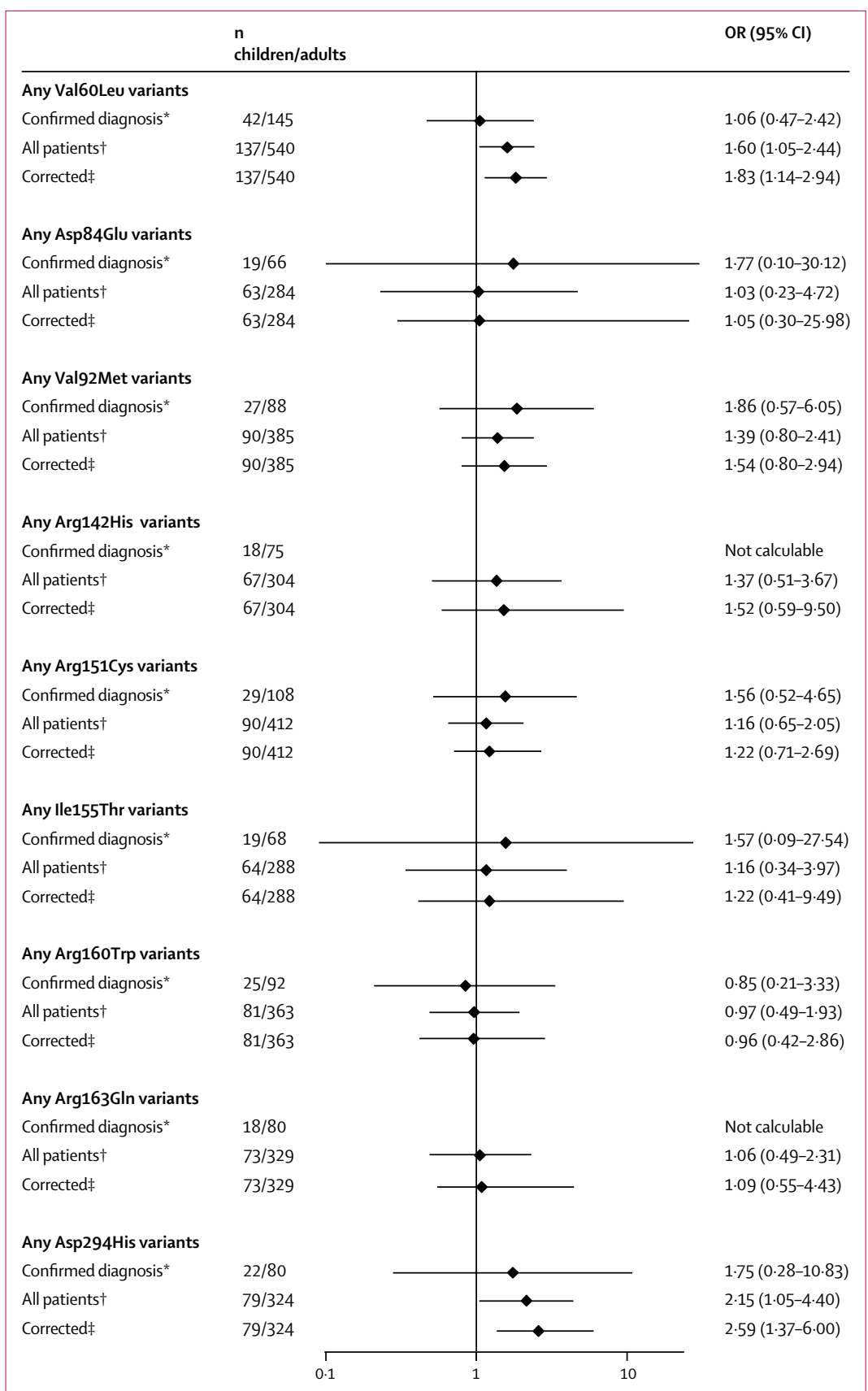

Figure 4: Covariable-adjusted odds ratio (OR) for the association between the nine most prevalent MC1R variants and childhood and adolescent melanoma compared with adult melanoma All ORs were adjusted by sex, matching stratum variable, melanoma body site and histopathological type, hair colour, and skin type. For each OR, the comparison groups included young patients matched (4:1) with adult patients by study or geographical area. The reference category for OR was MC1R wild-type (WT) individuals. Numbers of children or adolescents and adults reported here are the total numbers of patients included in each analysis, independently by MC1R status. For the analysis on each variant versus WT, patients carrying only other MC1R variants were excluded. R variants include Asp84Glu, Arg142His, Arg151Cys, lle155Thr, Arg160Trp, Asp294His, and other rare variants classified as R according to the algorithm proposed by Davies and colleagues. ${ }^{34}$ The r variants include Val60Leu, Val92Met, Arg163Gln, and other rare variants classified as r according to the algorithm proposed by Davies and colleagues. ${ }^{34} \mathrm{MC} 1 R=$ melanocortin 1 receptor. ${ }^{*}$ Calculated on the subgroup of patients with confirmed diagnosis of melanoma after centralised pathological review of glass slides. †Calculated on the whole sample of 233 young patients. ¥Corrected for probability of misdiagnosis, by combining information from OR of confirmed diagnoses and OR of all patients, as suggested elsewhere. ${ }^{35}$ 


\begin{tabular}{|c|c|c|c|c|}
\hline & \multicolumn{2}{|c|}{ Patients aged $\leq 18$ years $(n=148)$} & \multicolumn{2}{|c|}{ Patients aged $\leq 14$ years $(n=52)$} \\
\hline & $\begin{array}{l}\text { Young } \\
\text { patients/adult } \\
\text { patients }\end{array}$ & $\mathrm{OR}(95 \% \mathrm{Cl})$ & $\begin{array}{l}\text { Young } \\
\text { patients/adult } \\
\text { patients }\end{array}$ & OR $(95 \% \mathrm{Cl})$ \\
\hline Any variants & $148 / 592$ & $1.45(0.89-2.34)$ & $52 / 208$ & $1.86(0.69-5.03)$ \\
\hline Any $R$ variants & $73 / 330$ & $0.99(0.52-1.89)$ & $27 / 115$ & $1.63(0.42-6.36)$ \\
\hline Any r variants & $98 / 374$ & $1.80(1.06-3.07)$ & $35 / 142$ & $2.27(0.76-6.83)$ \\
\hline Any Val60Leu variants & $88 / 357$ & $1.59(0.91-2.76)$ & $31 / 136$ & $2.27(0.76-6.80)$ \\
\hline Any Asp84Glu variants & $43 / 192$ & $0.97(0.13-6.99)$ & $15 / 73$ & Not calculated \\
\hline Any Val92Met variants & $61 / 253$ & $1.62(0.79-3 \cdot 33)$ & $18 / 93$ & $0.95(0.11-7.97)$ \\
\hline Any Arg142His variants & $45 / 201$ & $1 \cdot 32(0.34-5 \cdot 13)$ & $13 / 80$ & Not calculated \\
\hline Any Arg151Cys variants & $56 / 277$ & $0.82(0.38-1.80)$ & $18 / 97$ & $0.61(0.10-3 \cdot 88)$ \\
\hline Any lle155Thr variants & $43 / 192$ & $1.13(0.17-7 \cdot 64)$ & $15 / 71$ & Not calculated \\
\hline Any Arg160Trp variants & $55 / 234$ & $1.08(0.45-2.58)$ & $21 / 87$ & $3.57(0.62-20.52)$ \\
\hline Any Arg163Gln variants & $51 / 219$ & $1 \cdot 61(0.61-4 \cdot 22)$ & $16 / 84$ & $0.68(0.03-14.81)$ \\
\hline Any Asp294His variants & $52 / 272$ & $1.47(0.58-3.70)$ & $14 / 86$ & Not calculated \\
\hline
\end{tabular}

Odds ratios (ORs) were adjusted by sex, matching stratum variable, melanoma body site and histological subtype, and skin type. Hair colour was not included because this category had more than $30 \%$ of missing data for these groups of patients. For each $\mathrm{OR}$, the comparison group included 4:1 frequency-matched adult patients by study or geographical area. The reference category for ORs were MC1R wild-type individuals. The number of children and adults reported here are the total number of patients included in each analysis, independently by MC1R status. For the analysis on each variant versus wild type, patients carrying only other MC1R variants were excluded. R variants include Asp84Glu, Arg142His, Arg151Cys, lle155Thr, Arg160Trp, Asp294His, and other rare variants classified as R according to the algorithm proposed by Davies et al..$^{34}$ The r variants include Val60Leu, Val92Met, Arg163Gln, and other rare variants classified as $r$ according to the algorithm proposed by Davies et al. ${ }^{34} \mathrm{MC} 1 \mathrm{R}=$ melanocortin 1 receptor.

Table 3: Subgroup analysis by age at diagnosis
Mediterranean area, where Val60Leu is the most frequent of all variants. ${ }^{40}$ The Asp294His variant is common in individuals with the red hair colour phenotype. The association of Asp294His with melanoma risk shows the heterogeneity between northern and southern European populations, where individuals who are more darkly pigmented are at higher risk of melanoma associated with Asp294His than are northern, less pigmented populations. ${ }^{41}$

To the best of our knowledge, our series of childhood and adolescent melanoma patients is the largest international multicentre cohort published so far with available $M C 1 R$ genetic data. The large number of young patients with melanoma, and comparable adult patients, provide powerful estimates of the association between $M C 1 R$ variants and childhood and adolescent melanoma within different populations. Another strength of our study was the centralised data quality control and statistical analysis that provided consistency across the numerous parent studies in defining and adjusting for important covariates. Histopathological centralised review of a third of the patients allowed us to calculate association estimates in a subset of children or adolescents with a histologically confirmed diagnosis and was helpful for calculating corrected risk estimates considering the issue of misdiagnosis.

Young patients with melanoma represent a heterogeneous group, including neonates, children, and adolescents, with various distinct presentations. ${ }^{9}$ Childhood melanoma might indeed differ from adolescent melanoma, and both might differ from adult melanoma. To further address heterogeneity between melanomas developed at different ages, we did a stratified analysis for patients aged 14 years or younger and 18 years or younger. Our non-significant findings from the younger subgroup might have resulted from decreased power related to the small sample size (59 patients) of this subgroup, whereas a separate multivariable analysis limited to children aged 10 years or younger was not possible because of the low number of patients (23 patients). In our child and adolescent sample, we had more darkly pigmented patients from southern European countries than from northern European origin, which might have resulted in high frequencies of $r$ variants, more common in southern Europe than in northern Europe. ${ }^{42}$ However, because young patients were compared with adult patients and controls from the same geographical areas, we do not believe this affected our results. Indeed, a sensitivity analysis done in the subgroup of young patients with adult patients sampled from the same parent study provided similar results. A centralised review of all melanomas would be desirable but, unfortunately, it was not feasible because of the retrospective nature of the study. To reduce disease misclassification, we excluded from the analysis patients whose histopathological reports were not available. We also provided risk estimates corrected for our observed 
misclassification prevalence among patients with histopathological centralised review, a group that was representative of the entire cohort of young patients. Nevertheless, we should note that this correction could not provide an exact estimate of the associations, as in a sample with only centrally confirmed diagnosed cases, and some imprecision of estimates could therefore not be ruled out. Because our cohort did not include patients with familial melanoma and the major susceptibility genes are rarely mutated in young patients, ${ }^{12,15,17,20}$ we did not analyse $C D K N 2 A$ and $C D K 4$ genes in our patients. It is possible that other major melanoma predisposition genes might influence the risk of disease in children and adolescents, but the absence of genetic data on these genes, such as BAP1, prevented the analysis of possible gene-gene interactions. Finally, although we did a high number of statistical tests, we allowed unadjusted $\mathrm{p}$ values to guide the interpretation of our results. Because of the exploratory, rather than confirmatory, nature of this study, we believe that our approach of describing the tests of significance we did, as advised by Thomas V Perneger, ${ }^{43}$ is appropriate. However, to directly address the issue of multiple testing, we also presented FDR-corrected $\mathrm{p}$ values.

In conclusion, our pooled analysis showed that natural variations in $M C 1 R$ are a genetic risk factor for childhood and adolescent cutaneous melanoma, as well as for adult cutaneous melanoma. MC1R variants, mainly $\mathrm{r}$ alleles, were suggested to have a greater role in childhood and adolescent melanoma than in adult melanoma, possibly through a pigmentation-independent pathway. Additionally, we observed a stronger effect of $r$ variants when the analysis was restricted to patients with melanoma aged 18 years or younger. Our study contributes to the comprehension of the genetic background of paediatric melanoma and elucidates the genetic diversity of paediatric and adult melanoma, with potential clinical relevance for developing early melanoma detection and preventive strategies.

\section{Contributors}

CP and SR did the literature search. SR, SG, PM, PAK, JCG-B, HN, MTL, JL, JN-B, FS, and MCF contributed to study design. M-FA, FD, BB-dP, VH, AEC, HA-C, SBG, RPG, LM, RZ, TD, NET, CBB, MB, SP MP, EN, PG, CMe, AMM, MR, SB, EP, LKS, FBa, GG, AS, FO, FA, RF-d-M, PQ, GR, AR, EM, IS, PAK, MAP, and MCF contributed to data collection. DM and FF did the histophatological review. CP and CMa did the molecular analysis of MC1R for new samples. FBo and SR analysed the data. CP, DM, SG, PAK, MTL, JL, MCF, and SR contributed to data interpretation. $\mathrm{CP}, \mathrm{FBo}$, and CMa wrote the manuscript. FB did the figures.

\section{M-SKIP Study Group}

Principal investigator: Sara Raimondi (IEO, European Institute of Oncology IRCCS, Milan, Italy). Advisory Committee members: Philippe Autier (International Prevention Research Institute, Lyon, France), Maria Concetta Fargnoli (University of L'Aquila, Italy), José C García-Borrón (University of Murcia, Spain), Jiali Han (Indiana University, Indianapolis, IN, USA), Peter A Kanetsky (Department of Cancer Epidemiology, H Lee Moffitt Cancer Center and Research Institute, Tampa, FL, USA), Maria Teresa Landi (National Cancer Institute, NIH, Bethesda, MD, USA), Julian Little (University of
Ottawa, Canada), Julia Newton-Bishop (University of Leeds, UK), Francesco Sera (London School of Hygiene \& Tropical Medicine, London, UK). Consultants: Saverio Caini (ISPO, Florence, Italy), Sara Gandini and Patrick Maisonneuve (IEO, European Institute of Oncology IRCCS, Milan, Italy). Participant investigators: Albert Hofman, Manfred Kayser, Fan Liu, Tamar Nijsten, and Andre G Uitterlinden (Erasmus MC University Medical Center, Rotterdam, Netherlands), Rajiv Kumar (German Cancer Research Center, Heidelberg, Germany), Tim Bishop and Faye Elliott (University of Leeds, Leeds, UK), Eduardo Nagore (Instituto Valenciano de Oncologia, Valencia, Spain), DeAnn Lazovich (Division of Epidemiology and Community Health, University of Minnesota, MN, USA), David Polsky (New York University School of Medicine, New York, NY, USA), Johan Hansson and Veronica Hoiom (Karolinska Institutet, Stockholm, Sweden), Paola Ghiorzo and Lorenza Pastorino (University of Genoa, Genoa, Italy), Nelleke A Gruis and Jan Nico Bouwes Bavinck (Leiden University Medical Center, Leiden, Netherlands), Ricardo Fernandez-de-Misa (Hospital Universitario Nuestra Señora de Candelaria, Santa Cruz de Tenerife, Spain), Paula Aguilera, Celia Badenas, Cristina Carrera, Pol Gimenez-Xavier, Josep Malvehy, Miriam Potrony, Susana Puig, Joan Anton Puig-Butille, and Gemma Tell-Marti (Hospital Clinic, IDIBAPS and CIBERER, Barcelona, Spain), Terence Dwyer (Murdoch Childrens Research Institute, VIC, Australia), Leigh Blizzard and Jennifer Cochrane (Menzies Institute for Medical Research, Hobart, Australia), Wojciech Branicki (Institute of Forensic Research, Krakow, Poland), Tadeusz Debniak (Pomeranian Medical University, Polabska, Poland), Niels Morling and Peter Johansen (University of Copenhagen, Denmark), Susan Mayne, Allen Bale, Brenda Cartmel, and Leah Ferrucci (Yale School of Public Health and Medicine, New Haven, CT, USA), Ruth Pfeiffer (National Cancer Institute, NIH, Bethesda, MD, USA), Giuseppe Palmieri (Istituto di Chimica Biomolecolare, CNR, Sassari, Italy), Gloria Ribas (Fundación Investigación Clínico de Valencia Instituto de Investigación Sanitaria- INCLIVA, Spain), Chiara Menin (Veneto Institute of Oncology IOV-IRCCS, Padua, Italy), Alexandros Stratigos and Katerina Kypreou (University of Athens, Andreas Sygros Hospital, Athens, Greece), Anne Bowcock,

Lynn Cornelius, and M Laurin Council (Washington University School of Medicine, St Louis, MO, USA), Tomonori Motokawa (POLA Chemical Industries, Yokohama, Japan), Sumiko Anno (Shibaura Institute of Technology, Tokyo, Japan), Per Helsing and Per Arne Andresen (Oslo University Hospital, Oslo, Norway), Gabriella Guida (University of Bari, Bari, Italy), Stefania Guida (University of Modena and Reggio Emilia, Modena, Italy), Terence H Wong (University of Edinburgh, Edinburgh, UK), and the GEM Study Group.

\section{GEM Study Group}

Coordinating Center, Memorial Sloan-Kettering Cancer Center, New York, NY, USA: Marianne Berwick (principal investigator, currently at the University of New Mexico), Colin Begg (co-principal investigator), Irene Orlow (co-investigator), Urvi Mujumdar (project coordinator), Amanda Hummer (biostatistician), Klaus Busam (dermatopathologist), Pampa Roy (laboratory technician), Rebecca Canchola (laboratory technician), Brian Clas (laboratory technician), Javiar Cotignola (laboratory technician), Yvette Monroe (interviewer). Study Centre, University of Sydney and The Cancer Council New South Wales, Sydney (Australia): Bruce Armstrong (principal investigator), Anne Kricker (co-principal investigator), Melisa Litchfield (study coordinator). Study centre, Menzies Institute for Medical Research, University of Tasmania, Hobart (Australia): Terence Dwyer (principal investigator), Paul Tucker (dermatopathologist), Nicola Stephens (study coordinator). Study centre, British Columbia Cancer Agency, Vancouver (Canada): Richard Gallagher (principal investigator), Teresa Switzer (coordinator). Study centre, Cancer Care Ontario, Toronto (Canada): Loraine Marrett (principal investigator), Beth Theis (co-investigator), Lynn From (dermatopathologist), Noori Chowdhury (coordinator), Louise Vanasse (coordinator), Mark Purdue (research officer). David Northrup (manager for CATI). Study centre, Centro per la Prevenzione Oncologia Torino, Turin (Italy): Roberto Zanetti (principal investigator), 
Stefano Rosso (data manager), Carlotta Sacerdote (coordinator). Study centre, University of California, Irvine (USA): Hoda Anton-Culver (principal investigator), Nancy Leighton (coordinator), Maureen Gildea (data manager). Study centre, University of Michigan, Ann Arbor (USA): Stephen Gruber (principal investigator), Joe Bonner (data manager), Joanne Jeter (coordinator). Study centre, New Jersey Department of Health and Senior Services, Trenton (USA): Judith Klotz (principal investigator), Homer Wilcox (co-principal investigator), Helen Weiss (coordinator). Study centre, University of North Carolina, Chapel Hill (USA): Robert Millikan (principal investigator), Nancy Thomas (co-investigator), Dianne Mattingly (coordinator), Jon Player (laboratory technician), Chiu-Kit Tse (data analyst). Study centre, University of Pennsylvania, Philadelphia, PA (USA): Timothy Rebbeck (principal investigator), Peter Kanetsky (co-investigator), Amy Walker (Laboratory technician), Saarene Panossian (laboratory technician). Consultants: Harvey Mohrenweiser, University of California, Irvine, Irvine, CA (USA); and Richard Setlow, Brookhaven National Laboratory, Upton, NY (USA).

\section{IMI study group}

Daniela Massi (University of Florence, Italy), Paola Ghiorzo and Lorenza Pastorino (University of Genoa, Italy), Chiara Menin (Veneto Institute of Oncology, IOV-IRCCS, Padua, Italy), Mauro Alaibac (University of Padua, Italy), Ausilia Maria Manganoni, Fabio Facchetti (University of Brescia, Italy), Monica Rodolfo, Andrea Ferrari, Barbara Valeri (Fondazione IRCCS Istituto Nazionale dei Tumori, Milan, Italy), Maria Concetta Fargnoli, Cristina Pellegrini (University of L'Aquila, Italy), Sonia Brugnara, Mariacristina Sicher, Daniela Mangiola (S. Chiara Hospital, Trento, Italy), Emanuela Passoni, Gianluca Nazzaro (Fondazione IRCCS Ca' Granda, Ospedale Maggiore Policlinico, Milan, Italy), Federica Baldini, Giulio Tosti, Sara Gandini, Giovanni Mazzarol (IEO, European Institute of Oncology IRCCS, Milan, Italy), Gabriella Guida, Stefania Guida, Giuseppe Giudice (Università degli Studi di Bari Aldo Moro, Bari, Italy), Fabrizio Ayala (National Cancer Institute, Fondazione G Pascale-IRCCS, Naples, Italy), Pietro Quaglino, Simone Ribero, Chiara Astrua (University of Torino, Turin, Italy), Antonella Romanini (Azienda Ospedaliero Universitaria Santa Chiara, Pisa, Italy), Emilia Migliano (San Gallicano Dermatological Institute, IRCCS, Rome, Italy), Ignazio Stanganelli, Laura Mazzoni (IRCCS-IRST Scientific Institute of Romagna for the Study and Treatment of Cancer, Meldola and University of Parma, Italy), and Maria Antonietta Pizzichetta (CRO Aviano National Cancer Institute, Italy).

\section{Declaration of interests}

We declare no competing interests.

\section{Acknowledgments}

We have received funding for this study from the Society for Pediatric Dermatology (SPD Pilot Project Award 2015) and Italian Association for Research on Cancer (AIRC MFAG 11831). PAK has received grants for the Melanoma Susceptibility Study from the National Cancer Institute (CA75434, CA80700, CA092428). PG has received funding for the Genoa study from the Italian Association for Research on Cancer (AIRC IG 15460). JL holds a tier 1 Canada Research Chair. The study in the Melanoma Unit, Hospital Clínic (Barcelona, Spain) was supported in part by grants from Fondo de Investigaciones Sanitarias (PI 12/00840, PI15/00956, and PI15/00716) and the CIBER de Enfermedades Raras of the Instituto de Salud Carlos III (Madrid, Spain); was co-funded by EU European Regional Development Funds and by the AGAUR 2014_SGR_603 and 2017_SGR_1134 of the Catalan Government (Spain); and was funded by a grant from Fundació La Marató de TV3 (201331-30; Catalonia, Spain), by the European Commission under the 6th Framework Programme (LSHC-CT-2006-018702; GenoMEL), by the CERCA ProgrammeGeneralitat de Catalunya, and by a research grant from the Fundación Científica de la Asociación Española Contra el Cáncer, Spain (GCB15152978SOEN). Part of the work was developed at the building Centro Esther Koplowitz, Barcelona (Spain). M-FA, FD, and BB-dP have received funding from the Hospital Programme for Clinical Research (France, PHRC 2007 AOM 07-195NI 07004). We thank the medical doctors who included some of the patients of this study. We wish to acknowledge the work of Gustave Roussy Biobank (BB-0033-00074) in providing DNA resources.

\section{References}

1 Nikolaou V, Stratigos AJ. Emerging trends in the epidemiology of melanoma. Br J Dermatol 2014; 170: 11-19.

2 Strouse JJ, Fears TR, Tucker MA, Wayne AS. Pediatric melanoma: risk factor and survival analysis of the surveillance, epidemiology and end results database. J Clin Oncol 2005; 23: 4735-41.

3 Austin MT, Xing Y, Hayes-Jordan AA, Lally KP, Cormier JN. Melanoma incidence rises for children and adolescents: an epidemiologic review of pediatric melanoma in the United States. J Pediatr Surg 2013; 48: 2207-13.

4 LaChance A, Shahriari M, Kerr PE, Grant-Kels JM. Melanoma: kids are not just little people. Clin Dermatol 2016; 34: 742-48.

5 Lorimer PD, White RL, Walsh K, et al. Pediatric and adolescent melanoma: a national cancer data base update. Ann Surg Oncol 2016; 23: 4058-66.

6 Steliarova-Foucher E, Colombet M, Ries LAG, et al. International incidence of childhood cancer, 2001-10: a population-based registry study. Lancet Oncol 2017; 18: 719-31.

7 Livestro DP, Kaine EM, Michaelson JS, et al. Melanoma in the young-differences and similarities with adult melanoma: a case-matched controlled analysis. Cancer 2007; 110: 614-24.

8 Berg P, Lindelöf B. Differences in malignant melanoma between children and adolescents. A 35-year epidemiological study. Arch Dermatol 1997; 133: 295-97.

9 Tracy ET, Aldrink JH. Pediatric melanoma. Semin Pediatr Surg 2016 25: 290-98.

10 Averbook BJ, Lee SJ, Delman KA, et al. Pediatric melanoma: analysis of an international registry. Cancer 2013 119: 4012-19.

11 Pappo AS. Melanoma in children and adolescents. Eur J Cancer 2003; 39: 2651-61.

12 Youl P, Aitken J, Hayward N, et al. Melanoma in adolescents: a case-control study of risk factors in Queensland, Australia. Int J Cancer 2002; 98: 92-98.

13 Whiteman DC, Valery P, McWhirter W, Green AC. Risk factors for childhood melanoma in Queensland, Australia. Int J Cancer 1997; 70: 26-31.

14 Ripperger T, Bielack SS, Borkhardt A, et al. Childhood cancer predisposition syndromes-a concise review and recommendations by the Cancer Predisposition Working Group of the Society for Pediatric Oncology and Hematology. Am J Med Genet A 2017; 173: 1017-37.

15 Berg P, Wennberg AM, Tuominen R, et al. Germline CDKN2A mutations are rare in child and adolescent cutaneous melanoma. Melanoma Res 2004; 14: 251-55.

16 Whiteman DC, Milligan A, Welch J, Green AC, Hayward NK Germline CDKN2A mutations in childhood melanoma. J Natl Cancer Inst 1997; 89: 1460.

17 Hocevar M, Avbelj M, Perić B, Zgajnar J, Besić N, Battelino T. High prevalence of germline CDKN2A mutations in Slovenian cutaneous malignant melanoma families. Croat Med J 2006; 47: 851-54.

18 Lu C, Zhang J, Nagahawatte P, et al. The genomic landscape of childhood and adolescent melanoma. J Invest Dermatol 2015; 135: 816-23.

19 Daniotti M, Ferrari A, Frigerio S. Cutaneous melanoma in childhood and adolescence shows frequent loss of INK4A and gain of KIT. J Invest Dermatol 2009; 129: 1759-68.

20 Rabbie R, Rashid M, Arance AM, et al. Genomic analysis and clinical management of adolescent cutaneous melanoma. Pigment Cell Melanoma Res 2017; 30: 307-16.

21 Wilmott JS, Johansson PA, Newell F, et al. Whole genome sequencing of melanomas in adolescent and young adults reveals distinct mutation landscapes and the potential role of germline variants in disease susceptibility. Int I Cancer 2019; 144: 1049-60.

22 Beaumont KA, Shekar SN, Newton RA, et al. Receptor function, dominant negative activity and phenotype correlations for $M C 1 R$ variant alleles. Hum Mol Genet 2007; 16: 2249-60.

23 Dessinioti C, Antoniou C, Katsambas A, Stratigos AJ. Melanocortin 1 receptor variants: functional role and pigmentary associations. Photochem Photobiol 2011; 87: 978-87.

24 Kanetsky PA, Rebbeck TR, Hummer AJ, et al. Population-based study of natural variation in the melanocortin-1 receptor gene and melanoma. Cancer Res 2006; 66: 9330-37. 
25 Pasquali E, García-Borrón JC, Fargnoli MC, et al. MC1R variants increased the risk of sporadic cutaneous melanoma in darker-pigmented Caucasians: a pooled-analysis from the M-SKIP project. Int J Cancer 2015; 136: 618-31.

26 Tagliabue E, Gandini S, Bellocco R, et al. MC1R variants as melanoma risk factor independent of at-risk phenotypic characteristics: a pooled-analysis from the M-SKIP project. Cancer Manag Res 2018; 10: 1143-54.

27 Fargnoli MC, Sera F, Suppa M, et al. Dermoscopic features of cutaneous melanoma are associated with clinical characteristics of patients and tumours and with MC1R genotype. $J$ Eur Acad Dermatol Venereol 2014; 28: 1768-75.

28 Vallone MG, Tell-Marti G, Potrony M, et al. Melanocortin 1 receptor (MC1R) polymorphisms' influence on size and dermoscopic features of nevi. Pigment Cell Melanoma Res 2018; 31: 39-50.

29 Robles-Espinoza CD, Roberts ND, Chen S, et al. Germline MC1R status influences somatic mutation burden in melanoma. Nat Commun 2016; 7: 12064.

30 Massi D, Tomasini C, Senetta R, et al. Atypical Spitz tumors in patients younger than 18 years. J Am Acad Dermatol 2015; 72: 37-46.

31 Elmore JG, Barnhill RL, Elder DE, et al. Pathologists' diagnosis of invasive melanoma and melanocytic proliferations: observer accuracy and reproducibility study. BMJ 2017; 357: j2813.

32 Busam KJ, Murali R, Pulitzer M, et al. Atypical spitzoid melanocytic tumors with positive sentinel lymph nodes in children and teenagers, and comparison with histologically unambiguous and lethal melanomas. Am J Surg Pathol 2009; 33: 1386-95.

33 Pellegrini C, Di Nardo L, Cipolloni G, et al. Heterogeneity of BRAF, NRAS, and TERT promoter mutational status in multiple melanomas and association with MC1R genotype: findings from molecular and immunohistochemical analysis. J Mol Diagn 2018; 20: $110-22$.
34 Davies JR, Randerson-Moor J, Kukalizch K, et al. Inherited variants in the MC1R gene and survival from cutaneous melanoma: a BioGenoMEL study. Pigment Cell Melanoma Res 2012; 25: 384-94.

35 Green MS. Use of predictive value to adjust relative risk estimates biased by misclassification of outcome status. Am J Epidemiol 1983; 117: 98-105.

36 Berk DR, LaBuz E, Dadras SS, et al. Melanoma and melanocytic tumors of uncertain malignant potential in children, adolescents and young adults— the Stanford experience 1995-2008. Pediatr Dermatol 2010; 27: 244-54.

37 Sanchez PC, Noda AY, Franco DD, Lourenço SV, Sangueza M, Neto CF. Melanoma in children, adolescents, and young adults: a clinical pathological study in a Brazilian population. Am J Dermatopathol 2014; 36: 620-28.

38 D'Mello SA, Finlay GJ, Baguley BC, Askarian-Amiri ME. Signaling pathways in melanogenesis. Int J Mol Sci 2016; 17: e1144.

39 Barón AE, Asdigian NL, Gonzalez V, et al. Interactions between ultraviolet light and MC1R and OCA2 variants are determinants of childhood nevus and freckle phenotypes. Cancer Epidemiol Biomarkers Prev 2014; 23: 2829-39.

40 Mills O, Messina JL. Pediatric melanoma: a review. Cancer Control 2009; 16: 225-33.

41 Gerstenblith MR, Goldstein AM, Fargnoli MC, Peris K, Landi MT. Comprehensive evaluation of allele frequency differences of MC1R variants across populations. Hum Mutat 2007; 28: 495-505.

42 Guida S, Bartolomeo N, Zanna PT, et al. Sporadic melanoma in south-eastern Italy: the impact of melanocortin 1 receptor (MC1R) polymorphism analysis in low-risk people and report of three novel variants. Arch Dermatol Res 2015; 307: 495-503.

43 Perneger TV. Adjusting for multiple testing in studies is less important than other concerns. BMJ 1999; 318: 1288. 